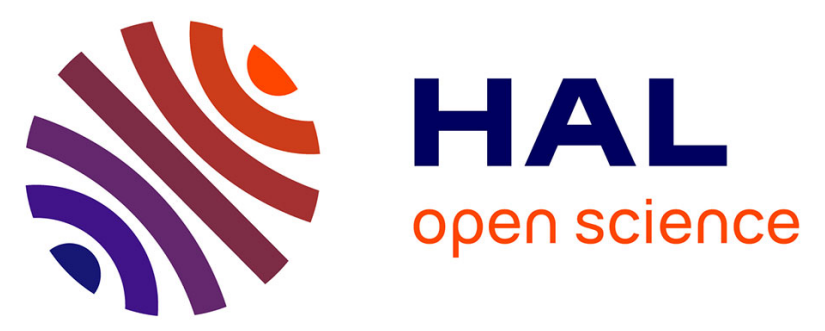

\title{
Pour une sociologie politique du supportérisme. Penser le militantisme et la partisanerie des supporters de football en Europe \\ Jean-Charles Basson, Ludovic Lestrelin
}

\section{- To cite this version:}

Jean-Charles Basson, Ludovic Lestrelin. Pour une sociologie politique du supportérisme. Penser le militantisme et la partisanerie des supporters de football en Europe. Thomas Busset; Roger Besson; Christophe Jaccoud. L'autre visage du supportérisme. Autorégulations, mobilisations collectives et mouvements sociaux, 6, Peter Lang, pp.21-40, 2014, (Savoirs sportifs), 978-3-0352-0267-0. 10.3726/978-3-0352-0267-0/11 . hal-02162730

\section{HAL Id: hal-02162730}

https://hal-normandie-univ.archives-ouvertes.fr/hal-02162730

Submitted on 22 Jun 2019

HAL is a multi-disciplinary open access archive for the deposit and dissemination of scientific research documents, whether they are published or not. The documents may come from teaching and research institutions in France or abroad, or from public or private research centers.
L'archive ouverte pluridisciplinaire HAL, est destinée au dépôt et à la diffusion de documents scientifiques de niveau recherche, publiés ou non, émanant des établissements d'enseignement et de recherche français ou étrangers, des laboratoires publics ou privés. 


\section{Pour une sociologie politique du supportérisme: penser le militantisme et la partisanerie des supporters de football en Europe}

Jean-Charles BASSON et Ludovic LeSTRELIN

Manifestations de rue, pétitions, prises de position dans le débat public, grèves des chants et des encouragements, création et développement de réseaux associatifs, lobbying tous azimuts, invocation de la défense des libertés publiques fondamentales, affirmation de revendications citoyennes, actions en justice... les mobilisations des supporters de football empruntent dorénavant au répertoire classique de l'action collective. Tant et si bien que nombreux sont les commentateurs de la scène ultra à succomber à la tentation de voir dans cette effervescence militante le ferment d'un renouveau démocratique propre à réformer nos vieilles sociétés occidentales $^{1}$. Sans verser dans la prophétie, il convient de prendre la mesure de la contribution du supportérisme au gouvernement des pratiques sociales d'une partie de la jeunesse européenne qui trouve là une opportunité, parmi d'autres, de se construire progressivement comme catégorie politique pertinente. Confrontant la sociologie de l'action publique (Hassenteufel 2008, Lascoumes et Le Galès 2007) de contrôle du supportérisme d'une part, à la sociologie de la mobilisation des supporters de football d'autre part, la proposition programmatique vise à dégager les conditions d'une sociologie politique des membres les plus fervents et les mieux organisés des tribunes des stades permettant de penser, par-delà l'usage métaphorique, le «militantisme» et la «partisanerie» des supporters en Europe (Bromberger 1995).

$\mathrm{Si}$, après une phase d'ajustement et de coordination de mesures diverses marquées par les disparités nationales, un ordre supérieur définit un espace public européen d'encadrement du supportérisme (1) (Basson 2004), une limite le caractérise: il mésestime les «bénéficiaires» auxquels il est des-

1 Associatif et militant, festif et démonstratif, indéfectible et radical, le supportérisme ultra est constitué de groupes de jeunes amateurs de football issus des classes moyennes et populaires se proclamant indépendants des clubs (Basson et Nuytens 2001). 
tiné. Considérés en tant que cibles, les groupes organisés de supporters sont, en effet, maintenus aux marges du processus d'élaboration et de mise en œuvre d'une action publique dont ils sont pourtant les premiers «ressortissants» (Warin 1999). Faisant valoir sa prétention à être considéré comme «lieu du politique» (2), le supportérisme ultra s'applique alors à user de l'originalité de son répertoire d'action collective afin de tenter de modifier les dispositifs d'action publique qui entravent son développement. Donner corps à notre proposition d'œuvrer à une sociologie politique du supportérisme suppose ainsi de mesurer «l'intérêt des monographies» (telle celle du Progetto ultrà de Bologne, en Italie) (3) propres à illustrer la dynamique du processus de légitimation politique du supportérisme.

\section{Un espace public européen d'encadrement du supportérisme}

Prenant appui sur un référentiel d'action publique partagé (Muller 2000), un espace public d'encadrement du supportérisme est en cours de codification à l'échelle européenne. Nul doute que la question

se présente différemment en fonction des lignes de fracture propres à chaque pays. En Angleterre, ce sont les inégalités, les disparités sociales et régionales qui comptent; en Ecosse et en Irlande du Nord, le sectarisme religieux; en Espagne, les nationalismes en partie fondés sur les différences linguistiques, entre catalans, castillans, galiciens et basques; en Italie, les particularismes liés aux villes et peut-être la division nord-sud [...]; enfin, en Allemagne, les relations entre les générations et celles entre l'est et l'ouest (Dunning 2010: 189-190)2.

Toutefois, un principe unificateur joue à plein: le propre du supporter étant d'accompagner «son» équipe dans tous ses déplacements (il est plus encore sollicité s'il réside «à distance» du club supporté, voir Lestrelin et al. 2006, Lestrelin et Basson 2009, Lestrelin 2010), et ainsi de franchir les frontières, l'hétérogénéité originelle des politiques publiques mises en œuvre par les pays concernés se voit réduite par l'imposition du caractère transnational du phénomène. Cette contrainte travaille à une forme

2 L'auteur ne s'encombre pas du cas français: caractère dérisoire (en dépit des drames récents) en regard des situations des pays voisins retenus ou difficulté à dégager des principes explicatifs à son sujet (Mignon 2002)? 
d'accommodation des actions engagées par les différents Etats et concourt à façonner un cadre d'interprétation légitime susceptible d'orienter l'action publique communautaire en ce domaine. Par le jeu de la porosité des cadres nationaux et de la convergence des finalités poursuivies par les Etats concernés, tous en viennent à adopter un corpus de normes et de dispositions relativement proches.

\section{La domination du modèle répressif et proactif développé en Angleterre}

De fait, ce processus de consolidation du référentiel européen présente des effets de distorsion. Le principal tient aux capacités des diverses institutions nationales à éprouver, dans leur contexte propre, de bonnes pratiques de gestion du supportérisme susceptibles d'être exportées vers les autres pays européens. Inégalement dotés, ces derniers voient le modèle, principalement répressif et proactif, développé en Angleterre assurer un rôle central dans la production du référentiel dominant. Incarnation du hooligan, la figure du supporter anglais vient comme justifier le dépassement des mesures traditionnelles de gestion des foules et de maintien de l'ordre et cautionner le recours aux options sécuritaires tendant au contrôle de la déviance (Tsoukala 2001 et 2010). Présenté comme une menace sociale, la répression du hooliganisme admet des entorses aux libertés publiques fondamentales et impose le déploiement de mesures inspirées des procédures de lutte contre les organisations terroristes et la grande criminalité. En retour, les dispositions radicales prises par les forces de l'ordre les cantonnent dans une opposition frontale avec les groupes de supporters et engendrent une surenchère entre deux parties tentées de s'engager dans une lutte sans fin. C'est ainsi que, face à des stades vécus comme des sanctuaires inviolables (Basson 2007a), les exactions se déplacent dans l'espace et le temps à la recherche de contextes propices à des manifestations de violence que l'intervention des forces de l'ordre ne viendrait pas interrompre: la pratique des fights (combats de rue ritualisés) organisés en marge des matchs de football, sur les aires d'autoroute ou au cœur des villes, se généralise. De même voit-on des groupes de supporters antagonistes s'allier pour organiser l'assaut des positions policières. L'actualité italienne (en Sicile et à Rome notamment) fournit des illustrations tragiques des effets de contournement du dispositif répressif mis en place (Lestrelin 2008). 


\section{Les limites du modèle sociopréventif développé en Belgique}

Si l'empreinte anglaise marque le processus d'européanisation de l'action publique de lutte contre le supportérisme, elle n'exclut pas d'autres modalités d'intervention. Ainsi la prévention offensive entend-elle s'attaquer aux racines sociales du problème (Comeron 2001) ${ }^{3}$. Elaboré en Belgique en réaction au drame du Heysel, le programme de fan coaching est imaginé au cours des années 1980 et généralisé à tous les clubs de football du Royaume. Il repose sur le constat que si le hooliganisme voit dans les tribunes des stades l'opportunité de se donner libre cours, il est le fait de supporters trouvant leurs motivations dans un autre espace-temps. C'est alors les facteurs sociaux du passage à l'acte qui sont pris en compte: situations familiales difficiles, scolarité défaillante, chômage persistant, habitat dégradé, perspectives individuelles sans lendemain... rendent nécessaires l'éducation, la responsabilisation, l'accompagnement et l'encadrement des supporters les plus dangereux. Appréhendé en tant que manifestation d'un dérèglement social, le hooliganisme appelle un mode de prise en charge total. Partageant leur vie, des éducateurs spécialisés assurent une présence quasi continue auprès des jeunes et s'appliquent à développer de nombreuses activités socioéducatives et socioculturelles susceptibles de canaliser leur énergie: rédaction et publication du fanzine (magazine du groupe), création et organisation d'un site électronique propre, gestion du fan home, «siège social» du groupe destiné à devenir le point de rencontre des acteurs publics et privés intéressés par le bon déroulement des matchs.

Ambitieuse, cette option sociopréventive affiche un bilan diversement apprécié. Les acteurs supportéristes insistent sur les limites et les contreparties qui affectent le dispositif: «Le fan coaching n'est pas une réussite [disent les supporters du Standard de Liège]. Ils veulent édulcorer l'essence même des ultras. [...] C'est un centre aéré pour adultes» (Rizzitelli et Jeannes 2008: 55). Les critiques touchent aux fondements du programme auquel il est reproché d'opérer le détournement et l'instrumentalisation du supportérisme. Sous le couvert de reconnaissance de l'engagement militant envers les équipes, le fan coaching serait une forme de contrôle réservé à une population qui échappe à l'encadrement social traditionnel. Plus encore, par sa forte dimension occupationnelle, il génèrerait la passivité des jeunes qui se comporteraient en consommateurs de spectacles sportifs et

3 La prévention situationnelle poursuit, quant à elle, d'autres objectifs (Basson, Le Noé et Diaz 2001, Landauer 2001). 
seraient ainsi maintenus dans une logique de paternalisme social. A cette ambiguïté originelle, viendrait s'ajouter une suspicion envers les éducateurs soupçonnés de servir indirectement les intérêts des services de police. Moins controversée, une dernière appréciation est partagée par les supporters et les acteurs publics en charge du programme: l'essoufflement, voire l'obsolescence, de ce dernier serait le fait du renouvellement conjugué des générations de supporters et d'éducateurs sociaux. A ce jour, le dispositif demeure uniquement à Charleroi et Liège (Fincoeur 2012).

\section{Le supportérisme ultra comme lieu du politique}

En vertu du principe selon lequel «l'action des destinataires (bénéficiaires) de la politique ou du programme est aussi structurante» (Lascoumes et Le Galès 2007: 36), les acteurs supportéristes entendent accéder aux réseaux publics afin d'y faire valoir leur répertoire d'action collective. L'ambition de pénétrer les arcanes de l'action publique en train de se faire repose sur l'affirmation des ressources proprement politiques dont disposent les groupes de supporters.

\section{Tribune(s) politique(s)}

Prendre leur part de la définition et de la mise en œuvre des politiques publiques qui leur sont destinées suppose, en premier lieu, de dépasser la reconnaissance de leur capacité à politiser, même de façon «limitée et sommaire» (Hourcade 2000: 110), les tribunes des stades. De même que Maurice Duverger notait que «la plupart des études relatives aux partis politiques s'attachent surtout à l'analyse de leurs doctrines» (1981: 19), les manifestations des idées et croyances, présentées comme rudimentaires et peu structurées, portées par la nébuleuse des associations supportéristes retiennent principalement l'attention des chercheurs. Ainsi les débats se focalisent-ils sur la situation respective des différents groupes sur un axe droite-gauche; sur l'infiltration et l'entrisme, réels ou supposés, dont certains sont l'objet ou sur l'usage et la signification de la rhétorique, des slogans, dénominations, chants, références, symboles, marques, 
emblèmes et signes cabalistiques les plus divers attestant la proximité, le ralliement, voire l'affiliation originelle, à telle ou telle ramification sulfureuse débordant le cadre des tribunes et les frontières nationales (Bodin et al. 2004: 57-67, Busset et al. 2008). Si cette lecture accorde une «fonction tribunitienne» (Lavau 1981) aux groupes supportéristes, elle repose sur une conception idéaliste de la politique entendue comme combat pour les grandes causes. Sont considérées à cette aune comme "politiques», les associations de supporters qui parviennent à dépasser les conflits d'intérêts particuliers (nécessairement corporatistes, voire triviaux et mesquins, telles les revendications de suppression des places assises dans les virages des stades ou de modération des tarifs des abonnements) et à s'inscrire dans la recherche d'une cause commune guidée par un mobile éthique témoignant d'une inclinaison pour les projets de société globaux, fussentils d'inspiration néofasciste. Or, le rapport de certains supporters «à la politique» et, plus fondamentalement, «au politique» semble dépasser l'emprunt, plus ou moins retravaillé, de figures et croyances idéologiques importées des cadres traditionnels.

\section{Les échelles de la participation politique des supporters}

Au-delà de ce premier relevé, pourquoi ne pas concéder aux groupes de supporters la capacité à s'organiser selon des principes politiques. Décrivant, dans un développement intitulé «la mobilisation», les membres du Commando ultra Curva B du stade de Naples comme des activistes modernes, professionnels et parfaitement organisés, Christian Bromberger ouvre la voie en ce sens (1995: 29-37). Son investigation du monde des «militants» supportéristes (selon le titre de son chapitre 14: 229-259) lui permet de dresser leurs caractéristiques essentielles: fidèles,

ils affichent des attitudes [...] avant-gardistes, cultivent un sens aigu de la spectacularité médiatique, organisent souvent leurs associations sur un mode managérial et recherchent, à travers leurs pratiques, tout autant à défendre une cause commune qu'à s'accomplir personnellement (Bromberger 1995: 241).

Mais il y a plus. Relevant qu'en «Italie et en France, les ultras forment fréquemment des associations rigoureusement structurées, disposant d'un local, distribuant des cartes d'adhérents, encaissant des cotisations, planifiant sourcilleusement la division des tâches militantes» (Bromberger 
1995: 242-243), son échelle de participation au groupe distinguant sympathisants, adhérents, militants et leaders renvoie à la taxinomie, classique en science politique, élaborée par Maurice Duverger pour classifier les structures organisationnelles des partis politiques (et des syndicats, Andolfatto et Labbé 2000: 71). Selon la typologie dressée par ce dernier, si les organisations officielles de supporters partagent de nombreux traits avec les «partis de cadres» (Basson et Nuytens 2001), les groupes organisés d'ultras peuvent être rapprochés des «partis de masse». Organisations centralisées prenant appui sur une cellule de base, ces derniers dessinent des «cercles concentriques» allant se resserrant et correspondant à quatre «degrés de participation» distincts qui comprennent, respectivement, les électeurs, les sympathisants, les adhérents et les militants. Du «spectateur-électeur» au «supporter-militant», en passant par l'«amateur-sympathisant» et l'«abonné-adhérent» (tel le socio espagnol), nul doute que les tribunes des stades présentent une large gamme de modalités de mobilisation renvoyant au monde du politique. Attester la diversité de la composition des publics des stades est une chose (Mignon 1995, Hourcade 2002: 80-83, Bodin 2003: 73-78, Nuytens 2004: 132-142, Bromberger et Lestrelin 2008: 126129, Hourcade 2010a: 164-165), dégager les différents degrés de participation structurant les organisations supportéristes en est une autre.

\section{Le supportérisme ultra comme mouvement social}

Reconnaître «qu'on ne se sait pas si l'état du supportérisme autonome dépend de facteurs organisationnels» implique de discuter l'assertion selon laquelle «le supportérisme indépendant ne s'accommode pas d'une division de l'engagement» et de récuser «qu'il ne soit pas question ici d'un mouvement social de type contestataire, d'une action collective structurée autour d'une situation conflictuelle» (Nuytens 2004: 229, 160 et 157). Ne pensant pas

pouvoir comparer le militantisme sportif [...] à d'autres participations individuelles classiques à l'action collective [...], comme celles d'agents des partis politiques ou d'acteurs réunis par exemple dans des collectifs ou dans des groupes contestataires (Nuytens 2004: 236),

la réticence à admettre le processus de qualification politique du supportérisme ultra s'explique aisément. Maintenu aux marges des arènes publiques 
au prétexte qu'il relève du domaine sportif conçu comme «un espace autonome doté de ses propres règles et de ses propres organes d'arbitrage entre intérêts divergents» (Defrance 2000: 26, Defrance 2001), il est entaché de l'affront qu'il adresse aux vertus cardinales prêtées au sport. «Une valeur durable de la culture sportive [étant] l'apolitisme» (Defrance 2000: 13), lui consentir l'apposition d'un sens politique revient, de fait, à remettre en cause la «volonté de résoudre l'antagonisme des classes par une réconciliation sur le terrain des loisirs» (Defrance 2000: 20). C'est ainsi qu'il est tentant de ne retenir que la dimension ludique et délassante du supportérisme et de «raisonnablement envisager de [le] penser en concurrence avec la pratique muséale, la visite d'autres sites culturels et touristiques, les promenades dans les parcs et les bases nautiques...» (Nuytens 2004: 160-161).

Le passage du supportérisme organisé au politique emprunte pourtant des formes traditionnelles ${ }^{4}$. Ainsi participe-t-il activement à l'alimentation du système politique. Il est, en premier lieu, un espace de socialisation politique et d'encadrement d'une jeunesse trouvant là l'occasion de se former aux rouages de la vie collective (Bromberger, Etienne et Guérin 2001). En effet, nombreux sont les exemples qui attestent qu'il est une instance d'éducation populaire, d'apprentissage de l'action collective, de construction d'une vision du monde, de façonnement d'une identité sociale, de partage d'expériences, de recrutement (par cooptation et parrainage), de sélection (par rites d'initiation et rites de passage) et de circulation d'élites militantes charismatiques investissant, à terme, les réseaux d'action publique. Il travaille également à l'agrégation des demandes et des revendications, à l'articulation des intérêts, à la mobilisation des soutiens ainsi qu'à la formulation publique et à la traduction politique des revendications d'une partie de la jeunesse; le tout à l'échelle européenne, territoire pertinent de la géographie politique du football, qui apparaît de plus en plus comme «le lieu où sont formulées et où s'affrontent les différentes qualifications des problèmes et où est définie la palette des solutions sur lesquelles les différents acteurs vont entrer en conflit ou en négociation» (Muller et Surel 1998: 99).

Le répertoire d'action collective des ultras européens comprend enfin des modalités de représentation, des formes de mobilisation, des registres d'expression et des schémas d'intervention renouvelés lui prêtant les

4 La participation active des supporters ultras sur certaines scènes du «printemps arabe» atteste que le processus n'est pas propre au cadre européen. 
caractéristiques d'un mouvement social à part entière 5 . Il constitue ainsi une forme originale des «nouveaux NMS» (Basson 2007b: 166-169). Nés dans le sillage de mai 1968, les «nouveaux mouvements sociaux» (NMS) sont dotés d'une forte aspiration identitaire et culturelle. S'inscrivant dans la relève du mouvement ouvrier (Touraine et al. 1984), ils se développent hors des milieux professionnels, débordent le répertoire des thèmes traditionnels, rassemblent (parfois de façon éphémère) des acteurs sociaux échappant en grande partie aux organisations partisanes et syndicales, n'obéissent pas aux modalités d'action de ces dernières et recourent à des formes singulières de mise en scène. Inspirés de ces derniers, la liste est longue des «nouveaux NMS» intervenant actuellement tant au niveau national qu'à l'échelle européenne et internationale (Crettiez et Sommier 2006). Luttant contre le racisme, prônant la défense de l'environnement, le développement durable ou la redistribution des ressources selon une vision altermondialiste (Agrikoliansky et al. 2005) ou «indignée», ils constituent des facteurs de transformation des modes routinisés d'engagement dans la sphère publique. Adoptant des formes réticulaires d'organisation, élargissant la portée des mobilisations jusqu'à l'échelle globale (Pleyers 2010), favorisant l'émergence de nouveaux acteurs, développant un usage intensif des nouvelles technologies de la communication (Granjon 2001), orchestrant la médiatisation de la dramaturgie des conflits, ils dessinent les contours d'un mouvement social publicisé et transnational d'un genre nouveau auquel le supportérisme ultra contribue par les ressources politiques qu'il recèle. Sa capacité à dépasser les revendications des tribunes pour les ouvrir à des causes générales (telles la valorisation de la culture populaire, la défense des libertés individuelles et la lutte contre les usages technologiques du contrôle social qui opèrent dans les stades), sa faculté à entretenir une forme de résistance à l'uniformisation et à la marchandisation de ce bien commun qu'est le sport (telles qu'illustrées par le spectacle mondialisé du football) en mettant en œuvre des manifestations festives hautement démonstratives et expressives, voire violentes et destructrices, prennent place dans l'espace public perçu comme un territoire à reconquérir.

Si ce mouvement social n'advient pas à ce jour c'est que le cadre d'interprétation dominant du supportérisme emprunte à une perception sociocognitive négative du phénomène. Représenté, nommé, qualifié et défini,

5 Sur l'analyse des transformations du système européen de représentation des intérêts, voir Balme et al. 2002, Greenwood et Aspinwall 1998. 
en un mot catégorisé, en tant que risque social spécifique, son inscription dans le débat public opère par la médiatisation des faits divers (l'usage des images et des témoignages est significatif), par la pratique de l'amalgame (avec hooliganisme, «violences urbaines» ou terrorisme) et par la surenchère sur son ampleur, ses ramifications et sa dangerosité (Hourcade 2010b, Lestrelin 2012). La sauvegarde de l'ordre public, la défense de l'intérêt général et la préservation de «l'éthique sportive» sont alors convoquées pour justifier une intervention radicale en vue de réprimer le phénomène. Une série de gestes symboliques et d'effets d'annonce accélèrent la mise sur agenda et autorisent le déploiement d'un large dispositif (réglementaire, législatif, judiciaire et policier) de répression du supportérisme tant à l'échelle nationale que communautaire et internationale. Disqualifiées par les modalités de construction du supportérisme en tant que problème public (Padioleau 1982), les organisations de supporters n'ont d'autre choix que s'interroger sur les conditions de la mise en politique de la cause supportériste qui, à ce jour, demeure «orpheline». La meilleure façon d'en rendre compte consiste à multiplier les investigations empiriques de terrain afin de restituer la «fibre militante» qui se donne à voir ici et là et l'ambition politique qui l'anime.

\section{De l'intérêt des monographies: le Progetto ultrà de Bologne (Italie)}

Explorer l'Europe [des supporters] «par le bas» [...] [revient à] en donner à voir la dynamique de construction dans sa profondeur et du point de vue des acteurs sociaux qui y sont confrontés ou qui en font usage (Pasquier et Weisbein 2004: 13).

A ce titre, le Progetto ultrà présente des ressources qui lui permettent de rejoindre le giron des «entreprises d'action collective» (Cefaï 2007: 26) mobilisées en ce domaine. Créée à Bologne en 1995, l'association invoque la déficience des politiques publiques nationales et européennes et se propose de les suppléer (Basson et Lestrelin 2008). Ainsi relève-t-elle que le processus d'européanisation de l'action publique tend à normaliser les modalités de prise en charge du supportérisme et en vient à nier les expériences nées de la prise en compte des singularités locales. A ce titre, 
le supportérisme ultra, majoritaire en Italie (Bromberger 1995, Roversi et Balestri 2000, Louis 2006), repose sur des pratiques associatives et militantes de régulation interne aux groupes de tifosi peu compatibles avec un référentiel d'action publique inspiré du principe de gestion des risques. Paradoxalement, la litanie des drames marquant l'actualité du football italien renforce cette conviction dans la mesure où l'atomisation du mouvement supportériste organisé est rendue responsable de la banalisation du recours à la violence. Dans ce contexte, le Progetto ultrà incarne un pôle de stabilité à même, d'une part, de filtrer et d'agréger les demandes et, d'autre part, d'assurer un mode d'expression et des débouchés aux revendications des supporters.

Rejetant un modèle répressif inadapté à la réalité italienne, les responsables associatifs de Bologne ne retiennent pas davantage les options sociopréventives promues par la Belgique. Ils insistent sur l'ambiguïté fondamentale qui les caractérise (Basson et Lestrelin 2008): «Pour moi, le fan coaching n'a rien à voir avec ce que nous faisons. En Belgique, ce n'est rien d'autre qu'un travail avec la police: il faudrait appeler ça du police coaching! Le travail social en pâtit. L'approche générale est suspecte!» explique Ashley Green, un des permanents. Le rejet du programme initié en Belgique s'accompagne d'une filiation symbolique à une mobilisation associative du même ordre originaire d'un autre pays européen. Universitaire cofondateur de l'association de Bologne, Carlo Balestri reconnaît que l'organisation italienne a pris le nom de Progetto ultrà en référence aux Fanprojekte allemands. Les responsables italiens prêtent deux vertus distinctives à ces organisations. Antérieures aux associations belges, elles sont libérées de l'emprise policière: «En Allemagne, un universitaire, un sociologue, un éducateur ou un supporter respecté peuvent être à la tête des Fanprojekte» spécifie Ashley Green. Dans les faits, le Progetto ultrà prône une «approche préventive proche du travail de rue et du social work» soucieuse d'éviter la stigmatisation et associant les groupes de supporters à l'élaboration et à la mise en œuvre des programmes qui leur sont destinés. Il s'agit, dit encore Ashley Green, «de respecter et de valoriser la culture populaire» dont ils sont porteurs. Ainsi les locaux de l'association font-ils office de centre de documentation sur les supporters et de conservatoire de la culture ultra présentant ouvrages et travaux universitaires, d'une part, et fanzines, écharpes, photographies, affiches, tracts, tambours ou encore bannières, d'autre part. 


\section{Expertise militante et citoyenneté ordinaire}

Ni cas sociaux, ni déviants, le Progetto ultrà entend considérer les supporters pour ce qu'ils sont et ne pas les inviter à la conversion. L'entreprise se garde des tentations normatives et compassionnelles et s'applique, dans le respect du droit, à préserver, affirmer et spécifier leur identité. L'association de Bologne propose ainsi d'opérer un changement de la perception publique du supportérisme et de rompre avec la logique de sauvegarde contre le risque qu'il incarnerait. De plus, le Progetto ultrà entend faire prévaloir que «les mobilisations collectives constituent des laboratoires d'action publique» (Cefaï 2007: 715). Si la citoyenneté ordinaire exprimée par les supporters et les savoirs profanes ne valent pas, de fait, expertises, ils contribuent à la construction de l'ordre social supportériste et orientent les conduites et les comportements. Maîtrisant ainsi les conditions sociales de mise en œuvre de l'action publique concernant les supporters, les cadres associatifs de Bologne aspirent à peser sur les actes de gouvernement. Espace non savant de production, d'accumulation, d'usages et de circulation de savoirs et de compétences spécifiques, le Progetto ultrà traduit, par un travail de transmutation, la contre-culture protestataire incarnée par les supporters en un levier original de modernisation de l'action publique (Robert 2008). Contribuant à la construction sociale du supportérisme en tant que problème public, l'ambition suppose d'attester un fort ancrage social et une connaissance intime du milieu ultra tout en affichant la capacité à s'en extraire pour être à même de soumettre la vulgate supportériste aux formes et aux exigences des politiques publiques. Réseau hybride, l'association italienne s'affiche tantôt comme un «observatoire social du supportérisme», tantôt comme la garde avancée d'un mouvement social critique et producteur d'une contre-expertise militante. Le Progetto ultrà est ainsi à même d'entrer en politique et de contribuer à bâtir des «coalitions de cause» (Sabatier et Jenkins-Smith 1993) dont le «militantisme des tribunes» est un des tenants.

«Partenaire social» de la négociation et «entrepreneur» de la cause supportériste, l'association italienne est à la recherche des «structures d'opportunités» lui permettant de faire aboutir ses options. Malaisée, la fonction de synthèse, de sélection et de transposition politique des mots d'ordre et des revendications des supporters est le propre de l'organisation italienne qui entend trouver là un gage de légitimité tant aux yeux des associations ultras que des pouvoirs publics. L'exercice est délicat tant le 
risque est grand de ne satisfaire ni les unes ni les autres et de se trouver discréditée par deux entités aux intérêts souvent inconciliables. L'expertise associative et militante du Progetto ultrà tient alors à sa capacité à rapprocher ou, si besoin, à arbitrer les différentes options en présence. A ce titre, son atout principal consiste à faire valoir que les expériences alternatives et les réalisations concrètes nées de la confrontation directe au terrain supportériste ne viennent pas concurrencer l'action publique mais compléter, voire pallier, des routines institutionnelles déficientes et générant des effets pervers. Testées localement, ces bonnes pratiques sont susceptibles de faire preuve d'adaptation à d'autres environnements et de se prêter à une forme de généralisation que les acteurs publics peuvent reprendre à leur compte pour réinvestir un espace social qui leur échappe grandement.

\section{Ennoblissement de la cause supportériste: l'antiracisme}

Couramment soupçonné de travailler à la traduction politique d'intérêts particuliers, le Progetto ultrà doit fournir la preuve que l'expertise dont il est porteur sert une large ambition. Il lui faut alors «monter en généralité» et attester que «les mouvements sociaux ne se limitent pas à des revendications particularistes, mais prétendent viser des biens qui sont profitables au plus grand nombre, sinon à tous» (Cefaï 2007: 15). Il trouve alors un motif d'ennoblissement de la cause supportériste dans son inscription dans la lutte contre le racisme. Chargée de projets à la direction nationale de l'Unione italiano sport per tutti (une fédération multisports à forte connotation éducative qui accueille en son sein les activités du Progetto ultrà), Daniela Conti déclare: «Le Progetto ultrà a deux principaux objectifs: lutter contre la violence et lutter contre le racisme. Etre antiraciste, ce n'est pas faire de la politique: ce sont les droits humains qui sont directement concernés!». Si l'argumentaire ne suffit pas à lever les critiques de certains groupes ultras reprochant à l'association son orientation socialisante, le supportérisme se voit ainsi reconnu cause générale de mobilisation par les buts ultimes qu'il poursuit. Qui plus est, il assure une fonction stratégique d'alerte publique.

Le problème du racisme est apparu dans le football avant que nous ne le rencontrions dans notre société. Les stades anticipent certains problèmes sociaux. Si vous êtes bien informés sur ce qui se passe dans les stades, vous êtes d'autant mieux préparés à gérer ces problèmes dans l'ensemble de la société 
poursuit Daniela Conti. Et d'égrener les nombreuses expériences relayées par le Progetto ultrà qui, sur l'ensemble du territoire italien, témoignent d'une forme d'intervention sociale avant-gardiste:

A Pérouse, ils ont entendu, en 2000, des cris de singe dans le stade. Ils ont distribué des tracts demandant aux spectateurs de couvrir ces manifestations racistes par des chants et des applaudissements. Après trois semaines, c'était fini! De même, les supporters de Modène proposent, en lien avec la municipalité, des projets antiracistes aux écoles et ont créé un centre de documentation sur ce thème. A Ancône, les supporters ont ouvert une hot line à destination des immigrés et font un gros travail social avec les différentes communautés de la ville. Enfin, il y a des expériences du même ordre à Empoli et à Pise, notamment...

La myriade d'initiatives développées localement, selon le réseau des groupes de supporters sensibles aux visées de l'association nationale, trouvent une forme de parachèvement, une fois l'an, à l'occasion de la tenue du Mondial antiraciste organisé depuis 1997 par le Progetto ultrà, assisté de l'Institut d'histoire de la résistance et des sociétés contemporaines. Evènement phare et ressource emblématique de l'organisation, le Mondiali antirazzisti autorise, tous les étés dans les environs de Bologne, la mise en scène des activités de l'association et permet d'afficher le rayonnement local, national et international de ses multiples partenaires, contacts et relais. "L'idée initiale était simple: rassembler des groupes de supporters et des communautés immigrées perçues comme criminogènes autour de matchs de football» explique Ashley Green. Accueillant plusieurs milliers de participants, la manifestation est devenue un véritable festival politico-culturel dont «le football n'est plus qu'un prétexte» (selon Ashley Green): débats, conférences, ateliers de réflexion, forums, concerts, présentation de livres et de vidéos, rencontres sportives, cuisine internationale, camping, fêtes... réunissent des supporters, des militants associatifs, syndicalistes et politiques venus de toute l'Europe.

\section{Conclusion}

Sous différentes formes et avec des succès divers, le Progetto ultrà italien, les Fanprojekte allemands, le fan coaching belge, le Fanarbeit suisse, 
mais également le Colectivo de prevención inserción social Andalucià de Cádiz en Espagne, le Svenska Fotbollssupporterunionen suédois, ou bien encore, à leurs manières, les socios des penyas espagnoles (abonnés au stade, adhérents du club, ils sont électeurs du président), la fédération Football Supporters Europe et les fans anglais (qui via Supporters Direct tentent de prendre une part minoritaire dans le capital de leur club en faillite) s'emploient à pénétrer les arènes publiques afin d'y faire prévaloir leur conception d'un football populaire en voie de disparition. Les militants français ne sont pas en reste: en janvier 2010, le premier congrès national des associations de supporters débouche sur la rédaction du Livre vert du supportérisme (Hourcade et al. 2010); l'association A la nantaise revendique le développement d'un actionnariat populaire inspiré du modèle anglais; le Collectif SOS Ligue 2 pétitionne contre le changement des horaires des matchs imposé par les télévisions sans tenir compte des disponibilités des spectateurs se rendant dans les stades... Le 13 octobre 2012, un millier de supporters ultras venus de toute la France défilent dans les rues de Montpellier pour dénoncer la «répression abusive» dont ils s'estiment victimes. «Notre passion: le football. Votre passion: le flashball» proclame la bannière des toulousains. A l'occasion d'une série de prises de parole précédant le départ de la manifestation, les organisateurs montpelliérains annoncent: «Nous sommes là pour revendiquer les droits des supporters, des ultras». Les responsables de l'association parisienne Liberté pour les abonnés précisent: «On est des supporters, on est des ultras, on est des passionnés; mais on est déjà des citoyens!».

Ces quelques exemples du vaste registre d'actions préventives que les organisations de supporters développent actuellement sont à même de contrarier les schémas d'intervention publique peu soucieux des «ressortissants» auxquels ils sont destinés. A ce titre, ils comptent parmi «les mobilisations associatives constitutives de la genèse d'une société civile européenne qui serait à la fois un contre-pouvoir aux institutions et une onction démocratique pour l'Europe» (Pasquier et Weisbein 2004: 9). Euvrer à une sociologie politique du supportérisme conjuguant une sociologie de l'action publique (Giraud et Warin 2008) et une sociologie des mobilisations (Fillieule et al. 2010), toutes deux sensibles à la mise au jour des fondements du processus démocratique contemporain, suppose investir ces différents terrains empiriques afin d'en mesurer la spécificité politique et, ce faisant, l'inclusion dans «l'espace des mouvements sociaux» (Mathieu 2007). 


\section{Bibliographie}

Agrikoliansky, E., Fillieule, O. et Mayer, N. (éds), 2005: L'altermondialisme en France. Genèse et dynamique d'un mouvement social, Paris: Flammarion.

Andolfatto, D. et Labbé, D., 2000: Sociologie des syndicats, Paris: La Découverte.

Balme, R., Chabanet, D. et Wright, V. (éds), 2002: L'action collective en Europe, Paris: Presses de Sciences po.

Basson, J.-C., 2004: «Les politiques de lutte contre le hooliganisme. Vers un référentiel européen d'action publique», In Roché, S. (éd), Réformer la police et la sécurité. Les nouvelles tendances en Europe et aux Etats-Unis, Paris: Odile Jacob, 313-330.

Basson, J.-C., 2007a: «L'espace du stade ou l'ordre en public», In Capron, G. et Haschar-Noé, N. (éds), L'espace public urbain: de l'objet au processus de construction, Toulouse: Presses universitaires du Mirail, 191-206.

Basson, J.-C., 2007b: «Vers une européanisation du syndicalisme français?», In Andolfatto, D. (éd), Les syndicats en France, Paris: La Documentation, 147-174.

Basson, J.-C., Le Noé, O. et Diaz, F., 2001: «La sécurité de la coupe du monde de football de 1998: bilan», In J.-C. Basson (éd), Sport et ordre public, Paris: La Documentation française, 175-188.

Basson, J.-C. et Lestrelin, L., 2008: «Vers une européanisation de l'action collective supportériste? Le Progetto ultrà de Bologne», In Tsoukala, A., Basson, J.-C., Lestrelin, L. et Sallé, L. (éds), Les enjeux des dispositifs actuels de lutte contre le hooliganisme en Europe, Rapport pour l'Institut national des hautes études de sécurité, Toulouse: L'Atelier de recherche sur les territoires et l'Europe, 175-202.

Basson, J.-C. et Nuytens, W., 2001: «Modes d'encadrement et de contrôle des supporters de football, entre modèle officiel et alternative autonome: le cas du Racing Club de Lens», Revue européenne de management du sport, 5: 1-29.

Bodin, D., 2003: Le hooliganisme, Paris: PUF.

Bodin, D., Robène, L. et Héas S., 2004: Sports et violences en Europe, Strasbourg: Editions du Conseil de l'Europe.

Bromberger, C., 1995: Le match de football. Ethnologie d'une passion partisane à Marseille, Naples et Turin, Paris: Maison des sciences de l'homme. 
Bromberger, C., Etienne, B. et Guérin M., 2002: «Les nouveaux lieux du politique», La Pensée du midi, 7: 79-91.

Bromberger, C. et Lestrelin, L., 2008: «Le sport et ses publics», In Arnaud, P., Attali, M. et Saint-Martin, J. (éds), Le sport en France, Paris: La Documentation française, 113-133.

Busset, T., Jaccoud, C., Dubey, J.-P. et Malatesta, D. (éds), 2008: Le football à l'épreuve de la violence et de l'extrémisme, Lausanne: Antipodes.

Cefaï, D., 2007: Pourquoi se mobilise-t-on? Les théories de l'action collective, Paris: La Découverte.

Comeron, M., 2001: «Pour une gestion sociopréventive du hooliganisme», In Basson, J.-C. (éd), Sport et ordre public, Paris: La Documentation française, 145-158.

Crettiez, X. et Sommier, I. (éds), 2006: La France rebelle. Tous les mouvements et acteurs de la contestation, Paris: Michalon.

Defrance, J., 2000: «La politique de l'apolitisme. Sur l'autonomisation du champ sportif», Politix, 50: 9-27.

Defrance, J., 2001: «Histoire du sport, régulations sociales et contrôle public. Entre autonomisation et mise sous tutelle», In Basson, J.-C. (éd), Sport et ordre public, Paris: La Documentation française, 9-19.

Dunning, E., 2010, «Approche figurationnelle du sport moderne. Réflexions sur le sport, la violence et la civilisation», Vingtième siècle. Revue d'histoire, 106: 177-191.

Duverger, M., 1981: Les partis politiques, Paris: Seuil.

Fillieule, O., Agrikoliansky, E. et Sommier, I. (éds), 2010: Penser les mouvements sociaux, Paris: La Découverte.

Fincoeur, B., 2012: «Les supporters face aux dispositifs de prévention de l'insécurité dans le football belge», Workshop L'autre visage du supportérisme: autorégulations, mobilisations collectives et mouvements sociaux, Centre international d'études du sport, Neuchâtel, Suisse, 14 septembre 2012.

Giraud, O. et Warin, P. (éds), 2008: Politiques publiques et démocratie, Paris: La Découverte.

Granjon, F., 2001, L'Internet militant. Mouvement social et usages des réseaux télématiques, Paris: Apogée.

Greenwood, J. et Aspinwall, M. (éds), 1998: Collective action in the European union, Londres: Routledge.

Hassenteufel, P., 2008: Sociologie politique: l'action publique, Paris: Armand Colin. 
Hourcade, N., 2000: «L'engagement politique des supporters ultras français», Politix, 50: 107-125.

Hourcade, N., 2002: «La place des supporters dans le monde du football», Pouvoirs, 101: 75-87.

Hourcade, N., 2010a: «Supporters extrêmes en France: dépasser les stéréotypes», Cahiers de la sécurité,11: 162-172.

Hourcade, N., 2010b: «Principes et problèmes de la politique de lutte contre le hooliganisme en France», Archives de politique criminelle, 32: 123-139.

Hourcade, N., Lestrelin, L. et Mignon, P., 2010: Le livre vert du supportérisme, Paris: Ministère de la santé et des sports.

Landauer, P., 2001: «La sécurisation des grandes enceintes sportives: la part de l'architecture. L'exemple du Stade de France», In Basson, J.-C. (éd), Sport et ordre public, Paris: La Documentation française, 189201.

Lascoumes, P. et Le Galès, P., 2007: Sociologie de l'action publique, Paris: Armand Colin.

Lavau, G., 1981: A quoi sert le Parti communiste français?, Paris: Fayard.

Lestrelin, L., 2008: «Ethnologie de la tifoseria de Rome. Violences, racisme et déstabilisation du supportérisme en Italie», In Tsoukala, A., Basson, J.-C., Lestrelin, L. et Sallé, L. (éds), Les enjeux des dispositifs actuels de lutte contre le hooliganisme en Europe, Rapport pour l'Institut national des hautes études de sécurité, Toulouse: L'Atelier de recherche sur les territoires et l'Europe, 93-138.

Lestrelin, L., 2010: L'autre public des matchs de football, Paris: EHESS.

Lestrelin, L., 2012: «Droit et radicalisation de la lutte contre le hooliganisme en France», In Guillaumé, J. et Dermit-Richard, N. (éds), Football et Droit, Paris: Librairie générale de droit et de jurisprudence-Fondation Varenne, 109-125.

Lestrelin, L. et Basson, J.-C., 2009: «Les territoires du football: 1'espace des 〈supporters à distance»», L'Espace géographique, 38 (4): 345-358.

Lestrelin, L., Sallé, L. et Basson, J.-C., 2006: «The trajectories leading to supporting at a distance: the Olympique de Marseille case study», European journal for sport and society, 3 (2): 125-141.

Louis, S., 2006: Le phénomène ultra en Italie. Historique du mouvement des groupes de supporters ultras de 1968 à 2005, Paris: Mare \& Martin. Mathieu, L., 2007: «L'espace des mouvements sociaux», Politix, 77: 131151. 
Mignon, P., 1995: «La violence dans les stades: supporters, ultras et hooligans», Les Cahiers de l'INSEP, 10: 13-36.

Mignon, P., 2002: «Une autre exception française: un football sans hooligans», Revue internationale de criminologie et de police scientifique, 55, (3): 323-347.

Muller, P., 2000: «L'analyse cognitive des politiques publiques: vers une sociologie politique de l'action publique», Revue française de science politique, 50 (2): 189-207.

Muller, P. et Surel, Y., 1998: L'analyse des politiques publiques, Paris: Montchrestien.

Nuytens, W., 2004: La popularité du football. Sociologie des supporters à Lens et à Lille, Arras: Artois Presses Université.

Padioleau, J.-G., 1982: L'Etat au concret, Paris: PUF.

Pasquier, R. et Weisbein, J., 2004: «L'Europe au microscope du local. Manifeste pour une sociologie politique de l'intégration communautaire», Politique européenne, 12: 5-21.

Pleyers, G., 2010: Alter-globalization. Becoming actors in the global age, Cambridge: Policy Press.

Rizzitelli, R. et Jeannes, D., 2008: «Bords de Meuse», So Foot, 51: 52-57.

Robert, C., 2008: «Expertise et action publique», In Borraz, O. et Guiraudon, V. (éds), Politiques publiques. 1. La France dans la gouvernance européenne, Paris: Presses de Sciences Po.

Roversi, A. et Balestri, C., 2000: «Italians ultras today: change or decline?», European journal on criminal policy and research, 8: 183-199.

Sabatier, P. et Jenkins-Smith, H. (éds), 1993: Policy change and learning: an advocacy coalition approach, Boulder: Westview press.

Touraine, A., Wieviorka, M. et Dubet, F., 1984: Le mouvement ouvrier, Paris: Fayard.

Tsoukala, A., 2001: «La gestion policière du hooliganisme: Angleterre, Italie, Pays-Bas», In Basson, J.-C. (éd), Sport et ordre public, Paris: La Documentation française, 159-174.

Tsoukala, A., 2010: Hooliganisme en Europe. Sécurité et libertés publiques, Montréal: Athéna.

Warin, P., 1999: «Les ressortissants dans les analyses des politiques publiques», Revue française de science politique, 49 (1): 103-120. 